\title{
Estuviste bien, Pirulo (tango, 1930)
}

\author{
El humor en un solo de bandoneón de \\ Pedro Maffia y el estilo bandoneonístico \\ de la década de $1920^{*}$
}

Omar García Brunelli

[Instituto Nacional de Musicología Carlos Vega]

Resumen Se analiza el tango Estuviste bien, Pirulo, compuesto en 1930 por S. Piana, con letra de R. Cordone y C. Goicochea. Como tango humorístico pertenece a una larga tradición de piezas que explotan una veta cómica. El yo lírico se encarna en una mujer que le reprocha a su pareja el abandono, en un tono irónico acentuado por el uso del lunfardo. De las tres versiones que se grabaron, una corresponde a A. Maizani y las otras dos al cantor R. Maida, una como estribillista de la orquesta de P. Maffia, y la otra como solista acompañado por la orquesta de A. Castellanos. Cuando el intérprete es el cantor, encontramos un mecanismo paródico adicional que no está presente en la versión femenina. Y en la versión de la orquesta de Maffia, además, el tono de parodia se extiende hasta la conformación misma del solo de bandoneón del director de la orquesta, que se estudia en detalle en el contexto de los solos y la función de los bandoneones en la orquesta típica entre 1923 y principios de la década de 1930, así como entre los solos del propio Maffia durante el mismo lapso.

Palabras clave: Tango humorístico · estilos bandoneonísticos 1920-1935 - Pedro Maffia
Summary This paper analyzes the tango Estuviste bien, Pirulo, composed in 1930 by S. Piana, with lyrics by R. Cordone and C. Goicochea. As a humorous tango it belongs to a long tradition of pieces that exploit a comic style. The lyric is about a woman who reproaches her partner for abandonment, in an ironic tone accentuated by the use of lunfardo. Among the three versions that were recorded, one corresponds to $A$. Maizani and the other two to the singer R. Maida, one as a chorus player in the P. Maffia orchestra, and the other as a soloist accompanied by the A. Castellanos orchestra. When the interpreter is the male singer, we find an additional parodic mechanism that is not present in the female version. And in the Maffia orchestra recording, moreover, the parody tone extends to the very composition of the bandoneon solo of the orchestra director, which is studied in detail in the context of the solos and the role of the bandoneons in the orquesta típica between 1923 and the early 1930s, as well as among Maffia's own solos during the same period.

Kew words: Tango and humor $\cdot$ bandoneon performing styles 1920-1935 · Pedro Maffia

* Agradezco el asesoramiento y consejos recibidos de mis colegas y amigos Romina Dezillio, Oscar Conde, Julio Schvartzman y Hernán Ramallo, para resolver algunas cuestiones durante la redacción de este trabajo. 
Me encontré por primera vez con este tango compuesto por Sebastián Piana, con letra de Rogelio Cordone y Carlos Goicochea ${ }^{1}$, en la versión grabada por Pedro Maffia y su orquesta en 1930. ${ }^{2}$ Me sorprendió el comentario hablado que formula una voz masculina grave, con un tono sarcástico: «Estuviste bien, Pirulo», luego de que el cantor Roberto Maida, aquí en calidad de estribillista ${ }^{3}$, canta el fragmento de la letra en el que una ex-milonguera se queja porque su galán, a quien le había estado "planchando los pantalones» durante los últimos diez años, la abandonó por otra milonga. La práctica del estribillista, de cantar solo una parte de la letra, o el estribillo, era común en la época, y según qué fragmento se cantara la comprensión del sentido del texto podía ser captada con mayor o menor precisión. ${ }^{4}$ El que canta Maida es el estribillo, que no alcanza para dar cuenta del significado cabal de la letra.

La felicitación a Pirulo resulta sorpresiva por el registro humorístico de la voz que la emite. También invoca al humor la incorrección política de felicitar, en una expresión coloquial sumamente popular en el habla rioplatense $\mathrm{e}^{5}$, al individuo que abandona a la mujer. $\mathrm{Al}$ ser un hombre el que le expide el juicio al varón que abandona, se estaría insertando un mecanismo humorístico adicional porque el cambio del estatuto de la voz varía el régimen del comentario: de cruel ironía a franca complicidad de género. ${ }^{6}$

1 El registro de autoría fue publicado en el Boletín Oficial nro. 56.583 del 8 de noviembre de 1931 y la fecha de registro en SADAIC fue el 28/6/1937.

2 Disco Columbia Record 6120. El registros corresponde a 1930, sin fecha exacta de grabación -no se especificaba en los libros de la discográfica-. Disponible en Youtube en https://youtu.be/OZUnfgf3LzO

3 Estribillistas o chansonniers se denominaba a los cantantes de las orquestas de tango a partir de mediados de los años veinte del siglo pasado, dado que solo cantaban el estribillo o segunda parte de cada tango (Ferrer, 1980: 1092).

4 En esta época ya el estándar de interpretación de un tango vocal-instrumental era ABAB para la música y ABCB para la letra (Vilariño, 1960: 40); si la letra se explayaba en alguna estrofa más, solía quedar fuera de la interpretación, causando a veces dificultades de comprensión o distorsión de sentido, como por ejemplo en el tango Marioneta (Romano, 2009: 38)

5 "Estuviste bien", acompañado del tono y la expresión indicada al decirlo, y en el contexto adecuado significa exactamente lo contrario. Se dice "estuviste bien" o "qué bien que estuviste" a alguien que nos perjudicó en algo. En ese sentido denota que alguien estuvo mal y nos hizo algún daño con su acción y expresa el reproche por la mala actitud. En el registro humorístico que aquí se le da se destaca su costado irónico.

6 Un estudio más amplio sobre los estereotipos de género en este tango excede los límites del presente trabajo. Para hacer más complejo el asunto, nótese que en las versiones de Maida, el cantor asume directamente el monólogo de una mujer. Aunque no es una circunstancia tan rara. Es posible hallar ejemplos de época en los que una cancionista expresa una voz masculina, pero con un marco introductorio (Sentencia, Pedro Maffia y Celedonio Flores, por Rosita 
La interpretación de Maffia tiene otro aspecto, que es el que más me interesa, y es el solo de bandoneón. Me resultó llamativo y poco corriente, realizado en un tono creciente y en escalas ascendentes hasta llegar a un clímax. La curiosidad me llevó a indagar un poco más sobre el tango.

Hallé que había sido grabado en otras dos ocasiones, una también por Roberto Maida, pero como solista, con la orquesta de Alberto Castellanos ${ }^{7}$; otra por Azucena Maizani con acompañamiento de piano y guitarra ${ }^{8}$, las dos en 1930 y en ambos casos se canta la letra completa.

A continuación transcribiré la letra a partir de la versión de Maizani ${ }^{9}$, señalando algunas pocas diferencias con la interpretación completa de Roberto Maida. ${ }^{10}$

Pirulo, jlindo servicio!

Te agradezco el sacrificio

de hacerme mujer decente.

Vos me sacaste del vicio

$\mathrm{pa}^{\prime}$ llevarme con tu gente.

Allí aprendí a laburar,

y en un ańo y otro y diez

Quiroga, 1926); un cantor dice un yo lírico femenino, también con marco (Lloró como una mujer, José María Aguilar y Celedonio Flores, por Carlos Gardel, 1929); o una mujer canta la voz de un hombre sin marco (El pechador, Ángel Villoldo, por Linda Thelma, 1909); y un cantor asume un yo femenino sin marco (Esa soy yo, de Adolfo Pérez y José Fernández, por Carlos Viván, 1929).

7 Disco Columbia Record № 6907. El registro corresponde a 1930. Es evidente la apuesta del sello discográfico por el tema, editando en forma simultánea las versiones de dos artistas reputados, Pedro Maffia y Roberto Maida, e incluso combinándolos en la grabación de Maffia. Se puede escuchar en https://youtu.be/KUxqlp980uk

8 Disco Brunswick 2123, con Manuel Parada en guitarra y Oreste Cúfaro en piano. Según la discografía de Gutiérrez Miglio, corresponde al año 1930. Los registros de Brunswick se realizaron entre 1929 y 1932 y en los libros del sello no se apuntaban las fechas exactas de la grabación. Se puede escuchar en https://youtu.be/ON2czY8-AR8

9 Puedo suponer que la versión inicial debe haber sido la de Maizani por ser la protagonista de la letra una mujer y por las cualidades histriónicas de la intérprete. Además Maizani tenía el antecedente de haber estrenado un tango importante de Piana, Silbando -en colaboración con González Castillo en la letra y Cátulo Castillo en la música-, en la revista Poker de Ases (1923) representada en el viejo Teatro San Martín. Es decir que había una relación profesional previa que habilita la suposición de que Piana le haya propuesto el tango a Maizani. Otra opción es que Cordone y Goicochea, los autores, gente de teatro, se lo propusieran a la cancionista. 0 tal vez Maizani hizo de nexo entre los autores y el compositor.

10 Por las versiones que se han grabado suponemos que debe haber sido una pieza de moda de relativo éxito, y por tanto debería haberse editado. Sin embargo, no he hallado un ejemplar en la Biblioteca Nacional ni en la Academia Nacional del Tango, ni en una buena cantidad de repositorios y coleccionistas que he consultado. Asimismo, las búsquedas en Internet fueron infructuosas. 
de zurcir y cocinar,

pude mi honor remendar,

y mina decente ser otra vez.

Los diez inviernos

de vida honrada,

de laburancia,

de privaciones,

y de plancharte

los pantalones,

hicieron de esta

«regenerada», ${ }^{11}$

una carreta

vieja y pesada,

con llantas llenas

de sabañones.

Y cuando ya me quitaste

toda chance 'e recaer,

me enteré que te piantaste

(¡Estuviste bien, Pirulo!) ${ }^{12}$

con otra milonga, ayer.

Pirulo, lindo servicio.

Te agradezco el sacrificio.

Te portaste a la più bella.

$\mathrm{Y}$ aunque como un desperdicio

me has echao tu parentela,

ahora ya puedo alternar

y gritar con altivez,

que es mi decencia ejemplar.

Pero, ¿¿dónde vi'á morfar

11 Maizani no canta la palabra "regenerada", como sí lo hace Maida. La dice de una forma muy expresiva, con un tono entre irónico y sarcástico.

12 En la versión de Maida este comentario está dicho por una voz masculina externa. Maizani lo dice hablando. 


\author{
(Batime, Pirulo) $)^{13}$ \\ con mi honradez? \\ ¡Si ni de serva \\ me dan laburo, \\ porque me encuentran \\ muy descolada! \\ Y si no salgo \\ de la ensartada \\ de aquel espiedo \\ que fue tu amuro, \\ es muy posible \\ que en la chivada \\ tome contacto \\ con el cianuro. \\ Y en la tapa de mi fosa ${ }^{14}$ \\ me pondrán esta oración: \\ «La mujer que aquí reposa ${ }^{15}$ \\ (Manyá qué dique, Pirulo) ${ }^{16}$ \\ regenerada crepó».
}

\title{
LA LETRA
}

Los autores eran expertos en calibrar el interés y la sensibilidad popular: fueron los exitosos y prolíficos Carlos Goicoechea y Rogelio Cordone, a quienes se les cuenta por lo menos veintiséis comedias entre 1923 y 1954.

13 Estos dos versos: "Pero, ¿dónde vi’á morfar / (Batime, Pirulo)" son hablados por Maizani con tono de tristeza. En cambio Maida los canta, escandiendo los versos con lógica musical y pericia: "Pero, ¿dónde vi’á morfar / (Batime, / Pirulo) con mi honradez." Es evidente que tienen línea musical asignada.

14 En la versión de Maida con Castellanos, Maida canta "en el mármol de mi fosa".

15 Este verso es cantado exageradamente por Maizani, remedando tal vez el estilo operístico y lo finaliza con una carcajada.

16 En la versión de Maida un tercero dice este verso. 
Varias de ellas fueron llevadas al cine ${ }^{17}$, medio para el que además colaboraron como guionistas, también en tono de comedia. Como autores de letras de tango no tuvieron una producción abundante. Con Sebastián Piana registran otro tango, $\mathrm{Pa}^{\text {qué lagrimear }}{ }^{18}$; con Pedro Maffia $N o$ te hagás la Greta Garbo ${ }^{19}$; y además colaboraron con la letra para cinco temas compuestos por Enrique Rodríguez - un vals, un tango, una milonga y dos foxtrots- que registró con su orquesta entre 1942 y $1944^{20}$, todos, salvo el tango, de tono festivo.

Cordone y Goicochea manejan con maestría la cantidad de lunfardismos que incluye la letra. Como explica Idea Vilariño (1960: 26-27), las letras de tango funcionan mejor con la incorporación de algunos vocablos lunfardos, pero si es en exceso atentan contra la credibilidad de lo que se dice. La letra incorpora quince lunfardismos. Para casi ninguno resulta necesario recurrir a un diccionario de lunfardo: laburar, mina, laburancia, te piantaste, milonga (apócope de milonguera: bailarina contratada en locales de diversión, también llamada copera), morfar, batí, serva (sirvienta, del italiano), laburo, ensartada (equivocación), amuro, chivada (enojo), manyá, dique, crepó. Y la deliciosa expresión «a la più bella», lunfardismo que mezcla español con italiano, con el significado «de la mejor manera posible».

17 Se realizaron filmes sobre sus obras Noches de Carnaval (1934) -film homónimo de 1938 dirigido por Julio Saraceni-; Boina blanca (1934) -film homónimo de Luis José Moglia Barth de 1941-; Canallita mío -Noche de bodas de 1942, dirigida por Carlos Hugo Christensen-; y La barra de la esquina (1950) -film del mismo nombre de 1950 dirigido por Julio Saraceni-. Además participaron como guionistas en El forastero (1937) dirigida por Antonio Ver Cioni y Segundos afuera! (1937) dirigida por Israel Chas de Cruz y Alberto Etchebere.

18 Pa' que lagrimear lo grabó Adolfo Carabelli en 1933, con estribillo cantado por Carlos Lafuente. Se puede escuchar en https://youtu.be/ZRBE5piXmUg

19 No te hagás la Greta Garbo lo grabó el propio Maffia con su orquesta en 1930, sin cantor. No he hallado la partitura pero se intuye la veta humorística a partir del título. Se puede escuchar en https://youtu.be/rvaolt8kOY4 20 Los temas, todos grabados por la orquesta de Enrique Rodríguez, son: Cómo has cambiado pebeta, tango (1942), cantado por Armando Moreno; los foxtrots Qué casualidad y Yo busco una novia, ambos de 1943, cantados por Armando Moreno; el vals Brindis (Despedida de soltero) (1944) cantado por Armando Moreno; y la milonga Juan Palomo, cantado por Ricardo Herrera y Fernando Reyes. 


\section{LAS INTERPRETACIONES DE MAIZANI Y MAIDA}

Azucena Maizani ${ }^{21}$ actúa la letra, más que simplemente cantarla con elocuencia y expresividad: cuando exclama «regenerada» en lugar de cantar, con una expresión de enojo o indignación; cuando dice (hablando) «Estuviste bien, Pirulo» y acentúa lo irónico de la expresión; cuando dice con tristeza «Pero, donde vi'á morfar? / Batime Pirulo», también hablado (Maida lo canta); y en el final, cuando canta "La mujer que aquí reposa» y remeda burlona un canto operístico o culto para acompañar irónicamente el lenguaje depurado del verso, seguido de una carcajada, para luego decirle a Pirulo «manyá qué dique» (mirá qué elegancia), y finalizar con enojo: «Regenerada crepó». La cancionista tenía una vasta experiencia escénica que evidentemente afianzaba el carácter de sus interpretaciones.

Por su parte, Roberto Maida ${ }^{22}$, en las dos versiones, la de letra incompleta con Maffia y la completa con Castellanos, realiza una versión perfecta, con un fraseo tanguístico impecable, sin ningún tipo de histrionismo adicional.

\section{...Pirulo como tango humorístico}

Me interesa simplemente destacar que ...Pirulo no es un caso aislado, se inserta en una larga tradición de tangos humorísticos o satíricos. Esta relación del tango con el humor ha sido estudiada por varios autores. ${ }^{23}$ En la bibliografía revisada se realizan varios inventarios abarcando un amplio rango de casos, desde Ángel Villoldo hasta Les Luthiers. Entre

21 Azucena Maizani (1902-1970) fue «acaso la más popular de las intérpretes femeninas del tango. Dueña de una voz sensitiva, de equilibrado vibrato y de un rico temperamento demarcó una época, creó un estilo vocal inconfundible que acompañó con la emoción propia de su presencia, capaz de llenar-por sí sola- un espectáculo.» (Ferrer, 1980: 647).

22 Roberto Maida (1908-1993) fue uno de los más destacados vocalistas entre 1925 y 1940, recordándose especialmente sus actuaciones con Francisco Canaro. Con posterioridad actuó con su propia orquesta y como solista (Gutiérrez Miglio, 1986: 2-9).

23 Por caso podemos mencionar los artículos de Eduardo Romano "El tango ríe» (2011: 109-128) y "Las letras de tango en la cultura popular argentina" (1983: 101). Juliana Guerrero se ocupa de un tango humorístico del repertorio de Les Luthiers. También Jorge Palacio (1996) trabajó sistemáticamente con este asunto. 
los ejemplos más notorios, cercanos en el tiempo al tango que estudiamos en este artículo, podemos citar La mina del Ford (1924), letra de Contursi y música de Antonio Scatasso y Fidel Del Negro; Victoria (1929) y Justo el 3 (I930) de Enrique Santos Discépolo; y iAtenti, pebeta! (1929) de Celedonio Flores con música de Ciriaco Ortiz.

\section{LA MÚSICA}

Dedico un breve párrafo al compositor, Sebastián Piana (1905-1994). De formación académica y popular, comenzó a componer a los diecisiete años admirando las ideas de Agustín Bardi y Juan Carlos Cobián. José González Castillo era amigo de la familia y con él compuso Sobre el pucho en 1922, su primer éxito, que Carlos Gardel grabó inmediatamente. Con el mismo autor y su hijo Cátulo Castillo compuso Silbando, muy exitoso (Kohan, 200I). En I930 conoció a Homero Manzi, y realizó con él una serie de colaboraciones de las que surgió una revitalización de la milonga, comenzando con Milonga sentimental. Posteriormente compuso obras de gran trascendencia; baste nombrar los tangos Tinta roja y El pescante, o el vals Caserón de tejas -las tres con letra de Cátulo Castillo-. En una época dirigió su Orquesta Típica Candombe ${ }^{24}$, y se dedicó a la docencia, pero fue fundamentalmente un compositor profesional de tangos, de los mejores de la historia del género.

Con respecto a Estuviste bien, Pirulo, su estructura atípica, como veremos, me inclina a pensar que el compositor trabajó sobre la letra ya realizada. Podemos imaginar diversos caminos por los cuales Cordone y Goicochea llegaron a Piana para proponerle el trabajo, como por ejemplo a través de Maizani, o por el vínculo del propio Piana con el mundo del teatro. ${ }^{25} \mathrm{La}$ música no presenta un gran interés per se; aunque permite que la letra se escanda de manera natural.

24 La Orquesta Típica Candombe tuvo escasa trascendencia. Con ella realizó unos pocos registros fonográficos entre 1940 y 1944. Posiblemente Piana quiso aprovechar el éxito de sus milongas, algunas de ellas con letras de temáticas vinculadas a la cultura porteña afrodescendiente.

25 Además del tango que le estrenara Azucena Maizani en una revista (ver nota 10) sabemos que también compuso con Cátulo Castillo la música para El ciego del violín (luego Viejo ciego), con letra de Homero Manzi, que fue estrenado 
Consta de dos secciones, una de quince compases (A) y otra de dieciocho compases (в). He realizado una transcripción a partir de la grabación de Maizani, que es la que supongo más cercana al original, sin respetar exactamente el fraseo de la cancionista y tomando algunos detalles de la versión completa de Maida, como para lograr una suerte de lead sheet que contempla solo la melodía (ver Imagen I). ${ }^{26}$ La frase inicial de A, cerrada, se dirige a establecer el tema (literario) del tango y ser el puntapié inicial de lo que sigue. A continuación la melodía se desarrolla por grado conjunto, en frases que van acompañando los versos. La música para: «Te agradezco el sacrificio / de hacerme mujer decente» se reitera con leves variaciones en los dos siguientes versos octosilábicos. Luego aplica una configuración melódica repetitiva para cuatro versos más. La repetición se interrumpe en el último, que es un endecasílabo, para el cual Piana escribe una frase conclusiva. Es evidente que la estructura resulta de seguir la lógica de los versos. No está planteada con la regularidad habitual de frases de cuatro compases y no se verifica la estructura de motivo con sus respectivos consecuentes que se determina para los tangos, en general, en el estudio estructural de la Antología del Tango Rioplatense (Ruiz y Ceñal, I980).

Para la sección в, los versos son pentasílabos. ${ }^{27}$ Piana le dedica un inciso a cada uno, en un esquema melorrítmico también repetitivo, de un compás, que solo se interrumpe luego de doce reiteraciones, cuando los versos pasan a ser octosílabos nuevamente. Entonces los resuelve con dos frases que abarcan cinco compases, que dejan espacio para la expresión hablada que se intercala («-Estuviste bien, Pirulo»). La monotonía se corta con una inflexión al relativo menor en la sexta frase, para regresar al Do Mayor original dos frases más adelante.

por Roberto Fugazot en Patadas y serenatas en el barrio de las latas (1926).

26 Los intercambios de información con Hernán Ramallo, así como sus consejos y criterios para definir detalles de esta transcripción, han sido sumamente provechosos.

27 Por lo menos, me permito considerarlos pentasílabos dado que la música se estructura en base a ellos. Los versos están en pares, y de unirlos, conformarían decasílabos. Pero al no contar con una partitura de editorial, no he podido constatar la intención de los autores. 
A
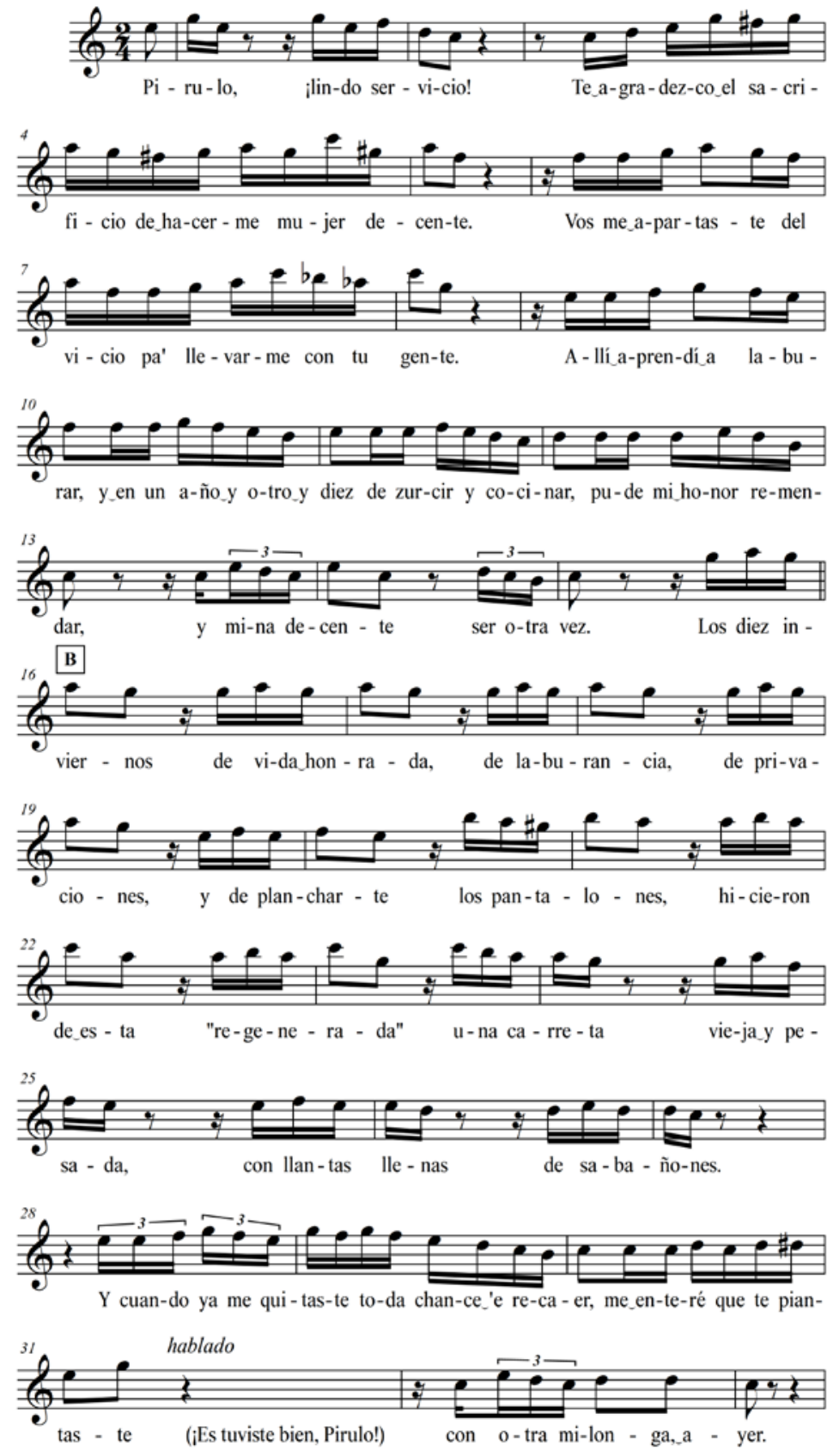

Imagen I: Transcripción de las dos secciones de Estuviste bien, Pirulo.

158 | Estuviste bien, Pirulo (tango, 1930) [OMar García Brunelli] 
Es evidente, luego de analizar la música, que pese a la corrección, lógica, y perfecto soporte para la letra que cumple la composición, el atractivo de la pieza es la letra.

\section{El SOLO DE MAFFIA}

Anticipo que el solo de bandoneón en el registro de Maffia con Maida es jocoso, según mi parecer, y que acompaña la comicidad general de la letra al remedar el discurso plañidero de la protagonista que expresa su queja. La línea del bandoneón va in crescendo hacia el agudo, como representando el enojo de la mujer.

Para poner este breve solo en contexto he recorrido sistemáticamente las grabaciones en las que participó el bandoneonista durante esos años, a las que me referiré enseguida. Pero antes explicaré qué representa Maffia para la interpretación instrumental del tango y en particular para la escuela bandoneonística.

Ferrer afirma que Maffia (I899-1967) se formó como bandoneonista en un momento en el que pudo conjugar dos influencias importantes: lo emocional de la interpretación de Eduardo Arolas (el bandoneonista más histriónico de la década de 1910) y la técnica del primer maestro sistemático del bandoneón, Arturo Bernstein (Ferrer, 1980: 635640) -aunque no estudió con este último-. Maffia logró su técnica aplicando la disciplina de ejercicios para ambas manos de los métodos de Hanon para piano, instrumento que había comenzado a estudiar antes que el bandoneón. A falta de maestros y métodos de bandoneón utilizó lo que tenía a mano. A su técnica sumó su personalidad interpretativa sobria, utilizando los recursos instrumentales que le daban expresividad y personalidad al tango de los años diez y veinte del siglo pasado. Su estilo se convirtió así en una de las vertientes, junto con las de Pedro Láurenz y Ciriaco Ortiz, que convergen en la modalidad bandoneonística de Aníbal Troilo, central en la historia del tango (Ferrer, 1980: 1039I040). Troilo resume esas influencias y se proyecta como canon hacia 
el futuro, alcanzando con su influjo hasta la madurez interpretativa de Piazzolla y más allá. ${ }^{28}$

Sabemos entonces que el estilo de Maffia resultó influyente. Su principal característica es una modalidad introvertida, de poco pero justo caudal sonoro, un fraseo muy sobrio, preferencia por el registro medio y utilización del registro agudo especialmente en puentes, arabescos (adornos) y variaciones. Manejaba a la perfección la técnica de apertura y cierre del fuelle del instrumento, usando las cuatro digitaciones sin solución de continuidad. ${ }^{29} \mathrm{Su}$ actitud era de mucha seriedad al interpretar, con poca movilidad corporal y un empleo moderado del fuelle en un rango de apertura y cierre muy estrecho. Su manera de tocar se puede observar en el corto Fuelle querido de Mauricio Berú ${ }^{30}$, en el que interpreta su tango Heliotropo. Zucchi (2007: 1366) le adjudica la invención del fraseo, de los arrastres, de los adornos. En realidad también suelen asignarse esas invenciones a Arolas -y tal vez a otros bandoneonistas-, por lo que no creo que sea posible hallar una autoría cierta sin revisar cronológicamente centenares de grabaciones, y de todos modos el resultado de la indagación sería confuso. Me inclino a pensar en una escena tanguística en la que unos pocos y grandes bandoneonistas se escuchaban entre sí aprovechando las invenciones de cada uno. Sin embargo, todos esos recursos se pueden hallar ya en los solos de Maffia desde las grabaciones de 1924 con el sexteto de De Caro, por lo que es muy posible que su estandarización y afianzamiento se deban en gran medida a él. Zucchi (2007:1367) no le atribuye, en cambio, la invención de las variaciones ${ }^{31}$,

28 Me refiero a la madurez interpretativa de Piazzolla que se puede observar con amplitud a partir de los largos solos que comenzó a desarrollar en su Noneto (1972-1974) y continuó luego en las obras del LP Libertango, de su etapa italiana (1974).

29 Como es sabido el bandoneón produce dos sonidos diferentes con la misma tecla según se expanda o se comprima el fuelle. Por lo tanto tiene una digitación distinta para cada mano según la dirección en que se produce la corriente de aire (aspirando o expulsando).

30 Fuelle Querido, corto de Mauricio Berú, se realizó en 1967 -disponible en https://youtu.be/jhOyfFfl4Z8-. La participación de Maffia en el corto comienza en 5'10".

31 Tomo la definición de "variación» del diccionario de Ferrer (1980: 1074): «Pasaje de notas agregadas y armonizadas contrapuntísticamente a la melodía natural de un tango (...) Se estructura como la melodía misma en uno o dos períodos de ocho compases cada uno". Desde el punto de vista técnico musical resulta más correcta la caracterización de Julián Peralta (2008: 45): «La variación es una técnica heredada de la música clásica. En el tango este tratamiento se basa en la densificación rítmica de la melodía, llevándola a un ritmo casi excluyente de semicorcheas (a veces es posible hallar algunas que incluyen seisillos $u$ otros valores irregulares). Usualmente la variación se 
pero sí su sistematización y difusión. León Benarós (1973) le preguntó una vez si él era el inventor de las variaciones y cuándo había empezado a utilizarlas. La respuesta fue la siguiente, y requiere cierta hermenéutica:

Allá por el año veinte me gustaba mucho hacer un contracanto cuando veía viable que era posible dentro del motivo de un tango. Como se utilizaban muchos violines para hacer armonía, yo pensé: el bandoneón podría también ser mañana un instrumento que exprese esa armonía y que puede quedar tan bien como el violín (Zucchi, 2007: 1367).

Maffia, con relación al origen, asimila variación a contracanto. Dice que se utilizaban mucho los violines para hacer «armonía» (a los contracantos de violín se les decía armonías de violín, no se está refiriendo al armado armónico vertical del arreglo) y que él pensaba que el bandoneón también podría realizarlas cuando lo consideraba oportuno. ${ }^{32}$ Aun así, resulta un tanto confuso que se refiera a las variaciones (que cuando se incluyen están en primer plano) como contracantos, que, por lo general, están en segundo plano. Sin embargo se comprende cabalmente lo que quiere decir cuando escuchamos en 2:I8 del tango La tranquera (Cipriano Nava) por el Sexteto de Julio De $\mathrm{Caro}^{33}$, en el que la melodía está a cargo de un bandoneón en el registro medio (Láurenz), mientras el otro bandoneón (Maffia) realiza una variación en el registro agudo, en segundo plano, como si fuera un contracanto.

Regreso ahora al solo de bandoneón en ...Pirulo. Reitero que tiene algunas particularidades con respecto al conjunto de solos de Maffia que escuché en mi recorrida por sus grabaciones de esa época fundamental del tango, cuyo resultado paso a resumir ahora.

construye como reexposición de una de las melodías del tango. Es común que se ubique hacia el final del arreglo cumpliendo, en muchos casos, la función de clímax." Ferrer (Ibíd.) afirma además que, por tradición oral, sabemos que las primeras fueron las que escribió e interpretó Arturo Bernstein alrededor de 1915. Entre las primeras, la más difundida fue la del tango Populacha (Romano y Celenza) -hay una excelente versión, justamente, por el sexteto de De Caro, supuestamente a cargo de Pedro Maffia; creo que es a ésta a la que se refiere Ferrer-. Finalmente, asegura que en la década de 1920, P. Maffia, P. Láurenz, E. Pollet y C. Marcucci, enriquecieron sus posibilidades.

32 Literalmente la respuesta de Maffia sería incomprensible. Seguramente hubo un problema en la transcripción de la entrevista, por falta de conocimiento específico.

33 Disco Victor 79548, grabado el 27/5/1925. Se puede escuchar en https://youtu.be/sfwkHiEGGp8 
En 1923 grabó en la orquesta de Juan Carlos Cobián -el otro bandoneonista era Luis Petrucelli, sin especificar prioridades-. Los registros fueron veintiséis, o sea trece discos, todos para el sello Victor. Desde I924 hasta noviembre de 1926 se desempeñó como bandoneonista en sesenta y cinco registros del sexteto de Julio De Caro. ${ }^{34}$ Luego organizó su propia orquesta con la que grabó ciento cincuenta registros entre 1929 y I936 (Lefcovich, 1980). ${ }^{35}$

Vamos ahora a lo que podemos escuchar de Maffia en esos registros. En las grabaciones de Cobián de I923 el dúo de bandoneones era, como afirmé anteriormente, Petrucelli y Maffia. ${ }^{36}$ Los otros integrantes de esta orquesta de Cobián eran los violinistas Agesilao Ferrazzano y Julio De Caro, luego De Caro con José Rosito de segundo violín, y el contrabajista Humberto Costanzo. Nos interesa la función que cumplían los bandoneones en los arreglos de los temas que se grabaron. En todos se desempeñan con permanente presencia en los acompañamientos, incluyendo numerosos arrastres en los comienzos de frase, en casi todos los temas. Participan con mucha frecuencia en breves puentes entre secciones o entre las frases de una sección, con breves y veloces escalas en el registro agudo (Una droga-Cobián-; Plata vieja-Rolla-). En algunos tangos alternan con el piano la presentación de la melodía (Polola-Rizzi-). Participan en arrastres realizados en tutti, seguidos de un silencio (No te olvides Durán-; La machona-José De Caro-; Adoración -Bianco-). A veces un bandoneón realiza un contracanto (El pirata -Nerón Ferrazano). ${ }^{37}$ Pocas veces toman la melodía ( La tipa -Enrique Maciel-). En ocasiones asumen finales de frase (Sea breve -Cobián-). El dúo de bandoneones puede comenzar una sección y alternar luego con otros instrumentos (Cacique-José Di Clemente-; Floreal-Paquita Bernardo-). No encontramos ningún solo extenso de bandoneón, ya que los arreglos involu-

34 En la primera grabación de la serie compartió el dúo de bandoneones con Luis Petrucelli y a partir de la segunda entró Pedro Láurenz en lugar de Petrucelli, siendo Maffia el primer bandoneón.

35 Hay algunas pocas y muy buenas grabaciones posteriores en las décadas de 1940, 1950 y 1960, pero no resultan relevantes para este trabajo.

36 Los bandoneonistas se habían encontrado por primera vez en la orquesta de José Martínez, en 1918, cuando tocaba en el cabaret L'abbaye. Se generó entre ellos inmediatamente una corriente de admiración y simpatía que los llevó a tocar juntos cuantas veces se diera la oportunidad (Zucchi, 2007: 1326-1327).

37 Este recurso se trasladó luego a la orquesta de Julio De Caro. 
cran la participación simultánea de los músicos casi por completo, con muy pocas partes solistas, y tampoco hallamos variaciones. Resulta virtualmente imposible saber cuál de los dos bandoneonistas toca en los breves fragmentos solistas de bandoneón.

Cobián abandona esta orquesta para viajar sorpresivamente a Estados unidos -el último tema grabado por el conjunto fue justamente Viaje al norte, de Cobián-. Los músicos se desbandan, pero Maffia y Petrucelli se las ingenian para tocar juntos en un par de lugares más (Zucchi, 2007: 1593).

A fines de 1923 los hermanos De Caro (Julio y Francisco) convocan A Maffia y Petrucelli a integrar un conjunto para tocar en las fiestas de la alta sociedad, muy bien pagados. Los bandoneonistas acceden con poco entusiasmo, según afirma Zucchi (2007:I335). Tal vez consideraran advenedizos a los jóvenes De Caro, aunque eran coetáneos que rondaban los 25 años (Petrucelli era 4 años menor). Creo que, seguramente, en la escena del tango pesaba más el prestigio bandoneonístico -ya eran dos protagonistas estelares- que el virtuosismo violinístico de De Caro. ${ }^{38} \mathrm{~A}$ principios de 1924 el nuevo grupo toca en los carnavales con la plantilla ampliada. Y luego, nuevamente como sexteto, actuaron en el Vogue's, que funcionaba en el Palais de Glace, con un contrato muy ventajoso que había conseguido Julio De Caro. ${ }^{39}$ El conjunto resultó exitoso y fue convocado enseguida para grabar. Lo constituían Julio, Francisco y Emilio de Caro (violín, piano y segundo violín), el dúo de bandoneonistas que nos ocupa, y en el bajo Leopoldo Thompson. Las etiquetas de los discos requerían que se mencionara un director y los músicos aceptaron que fuera Julio. Pero dado el éxito de los discos, los dueños del Vogue's colocaron de manera inconsulta el nombre de De Caro como director

38 En particular Pedro Maffia era famoso desde muy chico. Sus seguidores lo apodaban "El pibe de Flores" y fue común desde entonces que cuando las orquestas en las que participaba tocaban en ambientes populares, el público le pidiera que tocara solo (Zucchi, 2007: 1331). Años después la admiración por su desempeño solista continuaba, como se desprende del testimonio de la participación de la orquesta de Maffia en un concurso de 1933: "la nota de verdadero arte, la puso la zurda mágica de Pedro, en un sencillo fraseo de "La Maleva" que emocionó tanto al público como a los accidentales contrincantes" (Zucchi, 2007: 1350-51).

39 Con respecto a esa etapa inicial del nuevo sexteto, Maffia afirmó: «El sexteto no tenía nombre. Hacíamos todo de común acuerdo. Cambiando ideas y mezclando esfuerzos. Iniciamos así, por obra de todos, una nueva modalidad en el tango. Más depurada. Más musical. Más técnica. Pero eso sí, sin desvirtuar el alma del tango, melódica y rítmicamente (Zucchi, 2007: 1335). 
en la marquesina y eso ofendió a Maffia y Petrucelli, que se retiraron. Por eso Petrucelli participó solamente en la primera placa del sexteto de De Caro. ${ }^{40}$ No obstante Maffia se reintegró al conjunto para participar del jugoso cachet que había obtenido De Caro, pues había sufrido un gran revés en el juego.

Maffia permaneció en el sexteto de De Caro hasta noviembre de 1926, contando con Pedro Láurenz como segundo bandoneón. ${ }^{41}$ Se había formado así la dupla bandoneonística más famosa de la historia del tango, dentro de uno de los conjuntos que fueron la base de todo el tango renovador $^{42}$ hasta la actualidad. Este nuevo dúo de bandoneones tenía una dinámica interna diferente a la de Maffia-Petrucelli. Maffia era el primer bandoneón y Láurenz el segundo, por lo que la distribución de roles no era tan pareja, adoptando obviamente mayor protagonismo Maffia. Además la organización interna del conjunto cambió con respecto a la que se observaba en el sexteto de Cobián. De Caro permitió una mayor participación de los bandoneones, o la fomentó, o la dinámica de organización de los arreglos condujo a que la distribución de funciones solísticas fuera pareja entre todos los instrumentos, permitiendo así que se destacaran los bandoneones. En esta época no había aun arreglos escritos. Se trabajaba utilizando las partituras de editorial como particelle, a las que eventualmente se agregaban los contracantos o algún detalle. El piano formulaba su propia participación y el director, o el consenso ${ }^{43}$, dictaminaban el orden de la interpretación. Para los bandoneones, acoplarse a las líneas melódicas de la pieza o realizar un acompañamiento era bastante automático. En cambio cuando asumían algún segmento

40 Luis Petrucelli siguió luego una carrera exitosa en orquestas de primera línea. Dirigió su propio conjunto en 1926 y lo disolvió para ir con Canaro a Nueva York. En 1927 rearmó su orquesta en Buenos Aires, con la que grabó entre los años 1928 y 1931. En esos registros se observa la función conductora de su bandoneón y se aprecian sus grandes dotes de instrumentista. En 1933 se incorpora a la orquesta de Fresedo, a la que aún pertenecía al fallecer en 1941, muy joven, a los 37 años, luego de una breve enfermedad (Zucchi, 2007: 1398-1603). Tal vez por su corta vida su influjo en el tango no ha sido tan gravitante como el de sus grandes colegas bandoneonistas.

41 Zucchi (2007: 1339) afirma que la última participación de Maffia en las grabaciones del sexteto fue el 29/11/1926. El 24/11/1926 había sido la última sesión de dúo con Láurenz.

42 Ferrer afirma que a partir del sexteto de De Caro, en 1925, se definen dos bandos: tradicionalistas y renovadores (Ferrer, 1980: 215-217). En la bibliografía también se los suele denominar evolucionistas y tradicionalistas (Sierra, 1966: 64-65)

43 En el caso particular del sexteto de De Caro se dice que la responsabilidad de la organización de los arreglos recaía sobre Francisco De Caro, el pianista (Sierra, 1976: 1088-1097). 
solístico a dos bandoneones, la usual complejidad de ese pequeño subarreglo los llevaba a elaborarlos por separado, para luego incorporarlo en los ensayos al arreglo general. ${ }^{44}$ Este tipo de arreglos, realizados por «acuerdos previos» entre los músicos, y luego memorizados, fue la manera habitual de trabajo en muchos conjuntos como el de De Caro. ${ }^{45}$ Años más tarde, ante otros requerimientos, De Caro solicitó el concurso de arregladores profesionales, que fueron los primeros en incorporarse a la escena del tango reconocidamente en esas funciones.

La interpretación del dúo Maffia-Láurenz era interesante, compleja y muy tanguística a la vez. Su popularidad los llevó a que los convocaran a realizar diez registros fonográficos en dúo de bandoneones, para el sello Victor, entre 1925 y $1926 .{ }^{46}$ Podemos escuchar el desempeño del dúo en Pura maña (Maffia). ${ }^{47} \mathrm{El}$ ensamble es perfecto y el empaste tímbrico dificulta discernir quién toca cada cosa. Sin embargo aventuro que en o:34 Maffia toca la melodía y que Láurenz aparece en primer plano en I:22; y en 2:50 un bandoneón expone llanamente la melodía y el otro (creo que Maffia) hace una variación. Si bien el virtuosismo de ambos es parejo, la personalidad interpretativa de Láurenz siempre fue más expansiva e impetuosa y eso lo conducía a un fraseo más intenso.

En los sesenta y nueve registros del sexteto de De Caro en los que tocó Maffia (Lefcovich, 1979), encontramos la participación de los bandoneones en los puentes (interseccionales o intraseccionales) aunque con menos frecuencia que en el conjunto de Cobián. Son de tipo escalístico (Mosca Brava-D. Pereyra-). En la misma línea observamos escalas como anacrusas (upbeat) en el comienzo de las secciones (Ivette -Rocca

44 Literalmente, los bandoneonistas trabajaban solos esta parte del arreglo, según manifestó Jorge Argentino Fernández al autor. Fernández (1915-2002) fue un importante bandoneonista que cobró gran prestigio con su participación junto a Aníbal Troilo en el sexteto de Elvino Vardaro entre 1933 y 1937. Ferrer (1980: 400-401) los considera una de las mayores parejas de bandoneones de todos los tiempos.

45 En otro tipo de orquestas, como las "institucionales" -Orquesta Típica Víctor; Orquesta Típica Brunswick; Orquesta Típica Carabelli-, la dinámica podía ser menos especializada, y los bandoneones nunca alcanzan ese tipo de complejidad. En general dedicadas a las grabaciones, el régimen de ensayos y arreglos debía ser a la fuerza más estandarizado.

46 Maffia declaró: «Formamos un dúo de bandoneones que se hizo famoso. (...) Hicimos muchas cosas lindas. Abrimos, creo, una nueva etapa al bandoneón. Hacíamos arreglos especiales. Y llegamos a tener un afiatamiento realmente increíble." (Zucchi, 2007: 1338-1339).

47 Pura Maña (Pedro Maffia) por el dúo Maffia-Láurenz, disco Victor 79690 grabado el 7/7/1926 se puede escuchar en https://youtu.be/fDs1HvW6V9s 
y Costa-; La rayuela -De Caro-). Hay al menos tres variaciones, la más notable es la que aparece en Populacha (Celenza y Romano), ubicada en el sector central y no al final, y funcionando como un contracanto a la melodía principal; las otras dos están en La tranquera (Nava) y Pura maña (Maffia). Aparecen con bastante protagonismo los dúos de bandoneón integrados al arreglo, es decir con acompañamiento del resto del conjunto. Algunos presentan un fraseo poco acusado, como los de La revancha (Láurenz) y Mala senda (E. Wiurnos); en La rayuela (J. De Caro) hay un dúo sin acompañamiento -solo con efectos percusivos de los violines-, en un arreglo en el que participan mucho los bandoneones; otros dúos recurren a un fraseo más expresivo, apelando al efecto de rezongo ${ }^{48}$, como los de Chiquilina (Romanelli) y Obrerita (Russo). Finalmente, en cuanto a los solos de bandoneón ${ }^{49}$, suelen estar en el registro medio, con un fraseo recatado y elegante, de tono melancólico contenido, siempre en legato, con mediana intensidad y a tempo con la pieza -es inusualmente presto el de Coperito (Mondano)-, como ejemplos planteo los de Quince abriles (Dolardo), Feliz viaje (A. Maffia), y especialmente Agua corriente (J. Rodríguez); otros solos son más fraseados, como los de Fuiste (La Rocca) en el registro agudo, y Mosca brava (D. Pereyra). ${ }^{50}$

Por último, me referiré al estilo de Maffia como solista de su propio conjunto, donde era indudablemente el protagonista bandoneonístico. Forma su orquesta inmediatamente luego de dejar, en buenos términos, la de De Caro. ${ }^{51}$ Trabaja intensamente con su conjunto lo que

48 El rezongo se realiza con segundas menores.

49 Habiendo dos bandoneones, uno podría preguntarse si no fue Láurenz en alguna ocasión quien los realizó. En principio asumo que estaban a cargo del primer bandoneón. En segundo lugar he tratado de determinar las diferencias estilísticas entre uno y otro. Las propongo más arriba cuando me refiero al dúo que realizan en el tango Pura maña. Además, he analizado también el desempeño de los bandoneones luego de la partida de Maffia -no me refiero a eso en este trabajo para no extender más los prolegómenos necesarios para abordar la cuestión central, es decir, el solo de Estuviste bien, Pirulo-, y se notan diferencias estilísticas y de carácter, debidas al nuevo protagonismo de Láurenz. Entonces, si alguna participación solista tuvo Láurenz como compañero de Maffia, evidentemente no quiso ir más allá del estilo del primer bandoneón. Todas estas observaciones que formulo se refieren a Maffia. Cuando he tenido alguna duda en algún tango, no los he colocado como ejemplo.

$\mathbf{5 0}$ Invito al lector/a a satisfacer su eventual curiosidad y escuchar estos tangos que proporciono como ejemplos en YouTube o Spotify donde están disponibles.

51 Era una época de extraordinaria bonanza económica y había suficiente trabajo para todos los conjuntos, y más si se trataba de figuras prestigiosas como Maffia. De hecho, trabajó ininterrumpidamente (atravesando la crisis de 
resta de 1926 y en los años siguientes, pero solo comienza a grabar cuando se incorpora como artista al sello discográfico Brunswick, en 1929. Allí grabó setenta temas hasta 1930. Luego, entre ese año y el siguiente, registró setenta y seis piezas para Columbia. Entre todas esas grabaciones hay veintisiete que son valses, rancheras y hay algunas folklóricas zamba, bailecito, estilo-. Las piezas restantes son tangos, de los cuales he analizado una muestra de cuarenta. Con respecto al estilo bandoneonístico que se observa en ellas he arribado a las conclusiones que detallo a continuación.

Los bandoneones realizan puentes, tanto de tipo escalístico (Golondrina-Piana-; La mariposa-Maffia y Flores-), como en modo fraseado (Golondrina, No te hagás la Greta Garbo-Maffia-), pero no son muy frecuentes. Encontré, como variante, un relleno de modo escalístico a dos bandoneones en Lindo varón (Mónaco y Forte). Las variaciones son frecuentes: las encontramos por ejemplo en Sentimientos (Abati); Aquel mozo rana (Baranbino); y Golondrina (Piana). También realiza variaciones a dúo de bandoneones, como puede observarse en Pelele (Maffia) y Maldita (Rodio y Flores). Obviamente, cuando corresponde, los bandoneones se integran a la melodía, los acompañamientos y los tutti. Por último, encontramos solos realizados con gran "prolijidad» (sin la proliferación de yeites ${ }^{52}$ que «borronean» el discurso, que son muy idiomáticos del género) como en Un pobre borracho (Vettori) y Sentimientos (Abatti); solos sin acompańamiento de la orquesta (también en Sentimientos y en M'estás cansando-Petrone-); y solos fraseados, como el de Sangre bohemia (Tripoli y Brancatti) y el de Vos también saliste bueno.

Me detengo en este último porque despliega una serie de recursos que también encontramos en el solo de Estuviste bien, Pirulo: unas pocas notas octavadas ${ }^{53}$, algún pequeño rezongo (segundas menores simultáneas), y la melodía de la primera sección -que es muy simple-, fra-

1930) con diversos conjuntos y en lugares de primera línea hasta 1945 (Zucchi, 2007: 1339-1361).

52 Los yeites (denominación emic) son los recursos interpretativos propios del tango, como los mencionados rezongos.

53 El octavado es una acciacatura de octava, un recurso esencial del estilo bandoneonístico de Ciriaco Ortiz, que casi todos los bandoneonistas han adoptado, por lo menos los de escuela decareana, desde Maffia hasta Piazzolla y Leopoldo Federico. 
seada tanguísticamente, con el agregado, entre las notas principales de la melodía, que son bastante ornamentadas, de escalas cromáticas que, en figuraciones veloces, llevan de una nota de la melodía a la otra (técnicamente, lo que se denomina "disminuciones», que se emplean para adornar la melodía). ${ }^{54}$ Un solo de similares características se escucha en Una limosnita por el amor de Dios (Vázquez-Bastardi) en o:32. ${ }^{55}$

Llegamos, finalmente, al solo de ...Pirulo. Como hemos visto hasta ahora el estilo de Maffia, es serio, intimista. Solo o en dúo, poco fraseado o muy fraseado y con yeites, en ningún caso he hallado que trate de realizar algún efecto humorístico. Aunque debo reconocer que en Rezongón (Ermoli), hace honor al título del tango, y en una frase de cuatro compases, el dúo de bandoneones expone la melodía con un muy acentuado rezongo de bandoneones ${ }^{56}$, en una obvia alusión al título para un auditor con las competencias adecuadas-. Rezongo, lloro, son los nombres émicos con que se designan esos lamentos que tan bien maridan con la frecuente melancolía del tango y que con frecuencia se reflejan en las letras. ${ }^{57}$

La acciacatura de octava, también usada en la mayoría de los solos fraseados de bandoneón, y en cualquier línea bandoneonística que quiera poner una cuota mínima de sentimiento y prosapia tanguística, fue un recurso gestado y desarrollado por Ciriaco Ortiz. ${ }^{58}$ Quizá lo podamos ver también denotando el llanto en clave de sorna, como en la versión de Llorón (Maglio - Cadícamo) por la orquesta de Canaro con Ernesto Famá. En I:53 el bandoneón solista expone la melodía de la primera sección con abundantes octavados. ${ }^{59}$

54 Vos también saliste bueno (Mazzeo-Forte) está disponible en https://youtu.be/rqzckWvU8nY, el solo se escucha en 1:06.

55 Una limosnita por el amor de Dios se puede escuchar en https://youtu.be/zLLfm31mAHc

56 En https://youtu.be/bsoq-OeuZ3l se puede escuchar Rezongón. El rezongo aparece en 0:10 y en 2:11.

$\mathbf{5 7}$ La cualidad rezongona de los bandoneones se menciona en muchas letras de tango, como por ejemplo en De puro guapo (Rafael Iriarte - Juan Carlos Fernández Díaz) de 1927; Alma Tanguera (Carlos Marcucci - Rosendo Llurba), registrado en 1939; Cuando tallan los recuerdos (Rafael Rossi - Enrique Cadícamo), registrado en 1939.

58 El octavado es un recurso «muy característico del bandoneón que consisten en la anticipación de la nota principal mediante una octava superior o inferior [la más usual es la superior]. (...) Se puede tocar restándole valor a la nota anterior o prolongando la octava para que tome más importancia que la nota ornamentada. Esto ocurre principalmente en la melodía expresiva, donde este adorno se utiliza más frecuentemente» (WOLFF, 2018: 196).

59 En https://youtu.be/UCZhWV3gB7s se puede escuchar El Ilorón de Juan Maglio y Enrique Cadícamo. Agradezco a Julio Schvartzman quien me hizo notar esta versión. 
A continuación podemos ver una transcripción aproximada del solo de Maffia (Imagen 2) -la versión de Azucena Maizani que he tomado para la pautación del tango está en do Mayor; la de Maffia en Re Mayor-. Los rezongos están en las segundas menores (mi\#-fa) de los compases i9, 20, 2I y 22. Los octavados caen más notoriamente (no los incluí en la transcripción) en los primeros tiempos de los compases I6, I7, I8 y i9, pero se intuyen en otros lugares, casi como notas «fantasma». Esos yeites, más la repetición machacona de las mismas notas, que está en la melodía original (la-si-la-si-la; la-si-la-si-la), que Maffia reitera más aún, acentúa el efecto de letanía, que tal vez buscó provocar Piana. Maffia toca esas notas con los octavados y con bordadura y además agrega, entre nota y nota de la melodía original, escalas (disminuciones) que dan la idea de un parlato reiterativo. Ese estilo cuasi discursivo que imprime al solo, es el que permite la asociación del mismo con la queja de la protagonista. Incluso cabría imaginarse que los rezongos mi\#-fa que mencioné antes, así como las notas un poco más largas en las que se apoya el bandoneón antes de comenzar cada inciso, pueden corresponderse con una interjección, también típica del porteño, que es una especie de «Jé!» pero con $\mathrm{h}$ aspirada y una vocal difusa entre e y a, denotativo de indignación o estrilo, muy a tono con el carácter humorístico de la pieza.

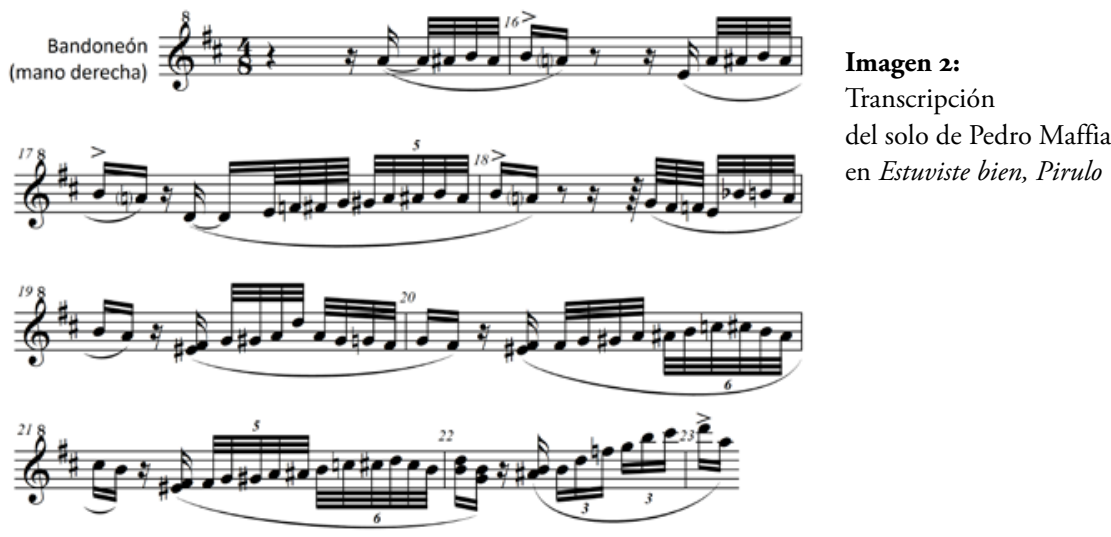


En este largo recorrido realizado para reflexionar en torno del solo de bandoneón de un tango olvidado, creo que he podido develar algunas cuestiones que inicialmente desconocía y pensar acerca de algunas hipótesis respecto de la circulación de los tangos alrededor de 1930.

También se ha puesto en evidencia el vínculo entre el mundo del tango y el mundo del teatro, a través de una escena musical y un ambiente teatral que se intersectaban con insistencia en las décadas de 1920, 1930 y 1940. Hemos visto un ejemplo más en el que suponemos (con bastante convencimiento) que la letra precedió a la música ${ }^{60} \mathrm{y}$ al adaptarse a los dictados de los versos, el compositor siguió más una lógica musical ad hoc que la norma formal establecida para el género (frases de cuatro compases para estructurar secciones de dieciséis compases). Para poner en contexto el solo de Maffia, debí estudiar sus prácticas bandoneonísticas desde 1923 hasta entrada la década de I930. Como siempre, la única forma posible de estudio es la inmersión en los registros fonográficos en forma cronológica, pues no hay aún un corpus de trabajos musicológicos que sirvan de base para discernir las prácticas instrumentales en el detalle que resulta necesario. Es cierto que los estilos orquestales en general están descriptos en la historiografía del tango, lo cual es de mucha ayuda, pero muchas veces los detalles están explicados de modo harto confuso por el manejo erróneo de conceptos musicales. También he debido incursionar en el subtema del tango y el humor, que amerita tal vez una atención más minuciosa que la recibida hasta ahora, involucrando preferentemente análisis musical. Constaté una vez más el valor del lunfardo en el buen desempeño de una letra de tango, y la precisión y lucidez de especialistas del tema, como Idea Vilariño, y Oscar Conde (2020). En todo este proceso, creo que lo más rico ha sido comprender el estilo de Maffia, y brindar el pequeño aporte de analizar un solo e indagar su trayectoria de instrumentista virtuoso, creador de un lenguaje bandoneonístico e intérprete genial de la prosapia tanguística.

60 Tema discutido largamente cuando analizamos tangos y no sabemos que es lo que se creó primero. El asunto es tratado brevemente por Pablo Kohan (2010: 20-22); incidentalmente por el autor, analizando obras vocales de Piazzolla (García Brunelli, 2014); y un poco más sistemáticamente por Julio Schvartzman (2015). 
Maffia podría haber adornado ...Pirulo con una variación de gran virtuosismo, o un solo fraseado como los que solía hacer, o un dúo con el segundo bandoneon, o bien con tan solo la melodía tal como es pero tocada de una manera expresiva. Sin embargo creo que eso hubiera sido superfluo. Su apelación a la parodia es superadora y devela una personalidad artística que va más allá del mero compromiso de mostrarse como primera figura, y capaz de entablar una cierta complicidad con el público, que sabía apreciar su pericia bandoneonística.

\section{REFERENCIAS BIBLIOGRÁFICAS}

BENARÓs, LEÓN (1973) «Entrevista a Maffia por León Benarós» Tanguera 1973,

(Zucchi, 2007: 1367).

FERRER, HORACIO (1980): El libro del tango. Crónica y diccionario, Buenos Aires, Tersol.

CONDE, OSCAR (2020): «El lunfardo em el repertorio de Carlos Gardel», en GARCíA BRUNELLI, Omar (compilador) El Mudo del tango. Ocho estudios sobre Carlos Gardel, Buenos Aires, Instituto Nacional de Musicología "Carlos Vega".

GARCIA BRUNELLI, OMAR (2014): "Los tangos con letra compuestos por Astor

Piazzolla entre 1945 y 1954" Avances, 23, Universidad Nacional de Córdoba,

Facultad de Filosofía y Humanidades, Centro de Investigaciones, Área de Artes. GUERRERo, JULIANA (2012) "Johann Sebastian Mastropiero: su incursión en el tango según Les Luthiers", en BUCH, Esteban: Tangos Cultos. Kagel, J.J. Castro, Mastropiero y otros cruces musicales, Buenos Aires. Gourmet Musical. GUTIÉRREZ MigLIO, ROBERTo (1986): “Vida y discografía de Roberto Maida», Cuadernos "Luces de tango", № 10.

KOHAN, PABLo (2001): «Piana, Sebastián», en Emilio Casares Rodizio: Diccionario de la música española e hispanoamericana (Madrid: Sgae, 752-753).

- (2010): Estudios sobre los estilos compositivos del tango (1920-1935),

Buenos Aires, Gourmet Musical.

LEFCOvich, s. Nicolás (1980): Estudio de la discografía de Pedro Maffia, Buenos Aires, el autor.

(1979): Estudio de la discografía de Julio De Caro (Buenos Aires: el autor. PALACIO, JORGE (Faruk) (1996): El humor en el tango, Buenos Aires, Corregidor. peralta, Julián (2008): La orquesta típica. Mecánica y aplicación de los fundamentos técnicos del tango, Buenos Aires, Edición del autor. 
ROMANO, EDUARDO (2009): “El tango y la literatura argentina» en LENCINA, Teresita; GARCÍA BRUNELLI, Omar y SALTON, Ricardo: Escritos sobre tango: en el Río de la Plata y en la Diáspora, Buenos Aires Centro 'feca. pp. 23-40. ROMANO, EDUARDO (1983): "Las letras de tango en la cultura popular argentina" en: Sobre poesía popular argentina, Buenos Aires, Centro Editor de América Latina.

(2011): «El tango ríe» en Teresita Lencina: Escritos sobre tango Vol. 2. Cultura rioplatense, patrimonio de la humanidad, Buenos Aires: centro'feca, pp. 109-128.

RUIZ, IRMA Y CEŃAL, NÉSTOR: "La estructura del tango", en Jorge Novati: Antología del Tango rioplatense, vol. 1, desde sus orígenes hasta 1920 (Buenos Aires: Instituto Nacional de Musicología "Carlos Vega", 1980). 2019 [1980].

schVartzman, julio (2015): El monstruo de la canción Revista Argentina de Musicología, 15-16.

SIERRA, LUIS ADOLFo (1966): Historia de la orquesta típica, Buenos Aires, Peña Lillo.

(1976): La escuela decareana, Buenos Aires, Corregidor.

VILARIŃo, IDEA (1965): Las letras de tango, Buenos Aires, Schapire.

WOLFF, EVA (2018): El bandoneón en el tango, Buenos Aires, Tango sin fin.

zUCCHI, OSCAR (2007): El tango, el bandoneón y sus intérpretes, Buenos Aires, Corregidor. 